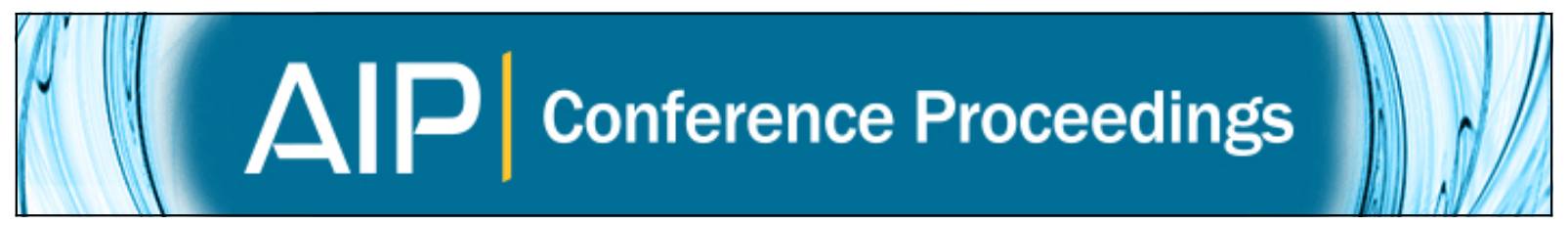

\title{
Experimental testing of various heat transfer structures in a flat plate thermal energy
} storage unit

Maike Johnson, Michael Fiß, and Torsten Klemm

Citation: AIP Conference Proceedings 1734, 050022 (2016); doi: 10.1063/1.4949120

View online: http://dx.doi.org/10.1063/1.4949120

View Table of Contents: http://scitation.aip.org/content/aip/proceeding/aipcp/1734?ver=pdfcov

Published by the AIP Publishing

\section{Articles you may be interested in}

Second test campaign of a pilot scale latent heat thermal energy storage - Durability and operational strategies AIP Conf. Proc. 1734, 050016 (2016); 10.1063/1.4949114

Heat transfer enhancement of a thermal storage unit consisting of a phase change material and nano-particles J. Renewable Sustainable Energy 4, 043124 (2012); 10.1063/1.4747824

Rotating flat plate condensation and heat transfer

AIP Conf. Proc. 246, 796 (1992); 10.1063/1.41877

Viscous and Joule Heating Effects on the Heat Transfer from a Flat Plate

Phys. Fluids 11, 437 (1968); 10.1063/1.1691924

Heat Transfer from a Flat Plate in Magnetohydrodynamics

Phys. Fluids 9, 2102 (1966); 10.1063/1.1761577 


\title{
Experimental Testing of Various Heat Transfer Structures in a Flat Plate Thermal Energy Storage Unit
}

\author{
Maike Johnson $^{1, \text { a) }}$, Michael Fiß ${ }^{1}$ and Torsten Klemm ${ }^{1}$ \\ ${ }^{1}$ German Aerospace Center (DLR), Pfaffenwaldring 38-40, 70569 Stuttgart, Germany \\ a) Corresponding author: maike.johnson@dlr.de
}

\begin{abstract}
For solar process heat applications with steam as the working fluid and varying application parameters, a novel latent heat storage concept has been developed using an adaptation of a flat plate heat exchanger as the storage concept. Since the pressure level in these applications usually does not exceed $30 \mathrm{bar}$, an adaptation with storage material chambers arranged between heat transfer medium chambers is possible. Phase change materials are used as the storage medium, so that the isothermal evaporation of steam during discharging of the storage is paired with the isothermal solidification of the storage material. Heat transfer structures can be inserted into the chambers to adjust the power level for a given application. By combining the required number of flat plate heat exchanger compartments and inserting the appropriate heat transfer structure, the design can easily be adjusted for the required power level and capacity for a specific application. Within this work, the technical feasibility of this concept is proven. The dependence of the operating characteristics on the geometry of the heat exchanger is identified. A focus is on varying the power density by integrating conductive heat structures in the PCM.
\end{abstract}

\section{INTRODUCTION}

Thermal energy storage is key for increasing energy efficiency and dispatchability, and latent heat storage is especially interesting for isothermal processes. Extended fin tube-and-shell storage concepts have been developed for solar thermal power plant applications [1] using thermally conductive aluminum in extended fins and for industrial applications using graphite fins [2] to counteract the low thermal conductivity of the phase change materials (PCM). These fin materials are interesting due to their compatibility with nitrate salts for high temperature applications, with graphite being limited to $250{ }^{\circ} \mathrm{C}$ and aluminum used for temperatures above this [3]. The design process for extended finned tubes in a tube-and-shell storage design requires an adaptation of the fin spacing and/or design and the tube pitch to adapt the storage for differing applications.

In applications for industrial processes, including processes with solar process heat supplies, a wider range of parameters are possible as system requirements - the media for charging and discharging, the temperatures, pressures and volumetric flow rates differ from process to process, in comparison to solar thermal power plant applications. In addition to this wider variation, the storage capacities are typically smaller than in solar thermal power plant applications, so that a large cost factor for storage unit integration remains the engineering of the storage unit and the system integration. In order to simplify the design and design process for storage units, an alternative latent heat storage concept has been developed [4]. Since the pressure level in these applications usually does not exceed 30 bar, the application of an easily adaptable flat plate thermal energy storage is possible. The PCM is filled between two neighboring flat plate heat exchanger compartments. The PCM compartments are open to the atmosphere at the top side of the storage. This has a dual purpose. On the one hand, the volume change that nitrate salts have during phase change is not problematic, as the salt is not contained in a closed container. On the other hand, an adaptation of a basic storage design can be achieved by inserting an appropriate heat transfer structure, thereby increasing the heat transfer surface area and the resulting power level achievable in the storage unit. First results of this work were published in [4]. In addition, in a design phase, the capacity of the storage unit can be quasi-modularly adapted through the concatenation of additional PCM compartments. As this adaptation is the 
simpler of the two, its analysis will be conducted in future studies. Within this work, the technical feasibility of the power level adaptation as well as the flat plate latent heat storage concept for high temperature phase change materials is proven.

\section{EXPERIMENTAL TESTING}

A storage unit with four PCM chambers around three flat plate heat exchangers with a reference surface area of $3.7 \mathrm{~m}^{2}$ was built. The design of this storage unit is reported in [4]. Thermal oil is used as the heat transfer fluid in the heat exchangers and a eutectic of $\mathrm{KNO}_{3}$ and $\mathrm{NaNO}_{3}(54: 46$ wt.\%) is the PCM. To avoid the addition of anti-caking agents into the storage, the eutectic was added as separate components to the storage unit. In-house measurements showed a melting temperature onset at $219.5^{\circ} \mathrm{C}$. This PCM has been used in several storage units, proving the reversibility of the phase change and stability of the composition in this temperature range. Further material properties are reported in [5] for the PCM and [4] for the storage unit and test rig. Approximately, the storage contains $250 \mathrm{~kg}$ of latent heat storage material, correlating to a latent heat of $7.5 \mathrm{kWh}$ and a nominal latent discharge time of 3.5 hours. A schematic of the storage unit tested is shown in Fig. 1 (a).

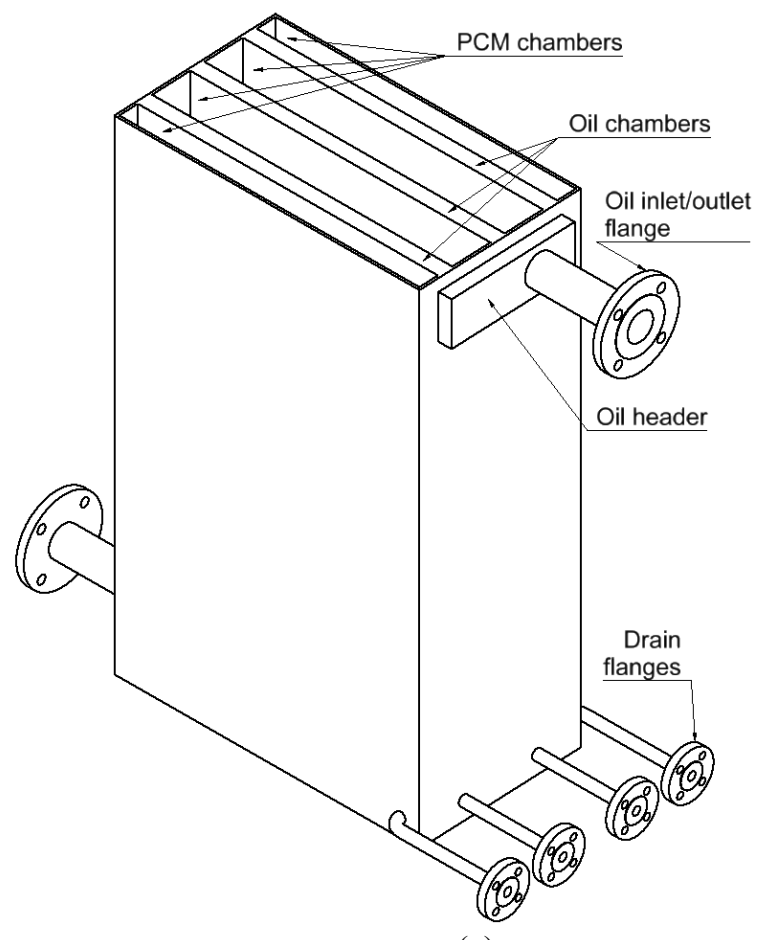

(a) (b)

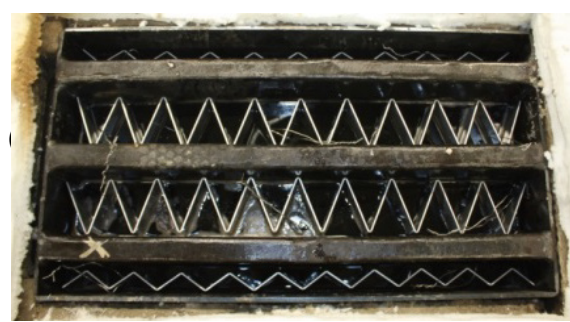

(c)

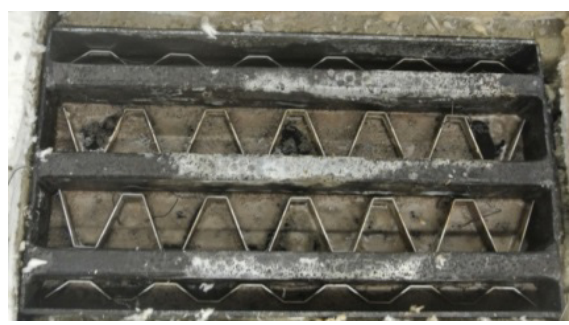

(d)

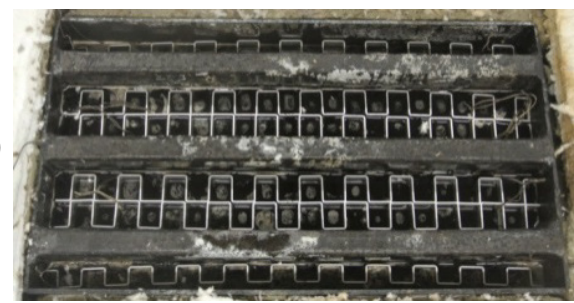

FIGURE 1: Schematic of the flat plat storage unit. Pictures of the (b) Zig-Zag, (c) Trapezoid and (d) Cross structures immersed in the charged storage unit.

Aluminum is selected for the conductive heat structure material due to its malleability and high thermal conductivity. The goal of the development of the heat transfer structures is to increase the power level of the storage unit while minimizing the amount of material used. The structures analyzed vary in their volume/mass and surface area. Each insertion of a structure displaces storage material. Therefore, a minimization of the structure volume is desirable. A large surface area, while maintaining contact with the heat transfer surfaces of the oil chambers, results in high power levels. Varying these aspects allows for an adaptation of the storage unit for application requirements.

Manufacturing aspects were considered in the identification of candidate geometries; structures with a Zig-Zag, a Trapezoidal and a Cross form were selected. Figure 1 (b, c, d) shows pictures of the structures immersed in the storage unit. Experiments at three temperature ranges about the melting point $( \pm 10 \mathrm{~K}, \pm 18 \mathrm{~K}$ and $\pm 25 \mathrm{~K})$ as well as at two volumetric flow rates $\left(0.5 \mathrm{~m}^{3} / \mathrm{h}\right.$ and $\left.2.0 \mathrm{~m} / \mathrm{h}\right)$ were conducted. Each experiment was repeated at least three times to ensure consistency in the results. 


\section{RESULTS AND DISCUSSION}

One of the benefits of latent heat storage is the possibility of having the heat transfer medium and the storage medium go through a phase change simultaneously, thereby keeping the temperature gradient in the storage unit constant and small throughout the charging and discharging cycles. In the test rigs available at DLR, however, thermal oil is the heat transfer medium. In order to simulate a phase change on the heat transfer side of the heat transfer, a high volumetric flow rate can be used. This results in nearly no temperature change from inlet to outlet of the storage unit and therefore nearly isothermal conditions, as would occur during phase change. For this reason, the results at the higher flow rate are given more attention. Excerpts of the results without heat transfer structures (HTS) and the initial results with the Zig-Zag structures were reported in [4]. In this paper, the results for all three HTS are compared to experiments without any HTS.

Figure 2 shows a comparison of the experimental results for one discharge cycle for each of the three structures as well as measurements with no structure in the storage unit. In these experiments, a volumetric flow rate of $2.0 \mathrm{~m}^{3} / \mathrm{h}$ was used. In Fig. 2 (a), the temperatures during discharge are shown for a temperature range about the melting point of $\pm 18 \mathrm{~K}$. The thermocouple measurements plotted here over time were located horizontally and vertically in the middle of one of the two large PCM chambers. In each case, the thermocouple measurement furthest from any HTS was selected, so that this measurement shows when the PCM furthest from a HTS or from environmental losses solidifies. This does not guarantee that all of the PCM in the chamber is solidified at this time, especially not the PCM vertically above this point. However, measurements at higher levels are complicated to track, since the volume change results in the thermocouples not always being immersed in salt, and the influence of thermal losses to the atmosphere, through the insulation, also affect the measurements. These curves all show a plateau as the PCM undergoes the phase change from liquid to solid and releases energy to the thermal oil. The change in temperature at the beginning of discharging is very fast, due to the large temperature difference between the molten salt and the heat transfer medium, the natural convection in the molten salt, and the optimal contact between the salt, the heat transfer surfaces and the thermocouple itself. After the PCM has solidified, there are various effects that result in the flatter slope of the temperature curve. On the one hand, there is no natural convection, reducing the heat transfer. On the other hand, the volume change in the salt during solidification occurs in all directions, so that not only is the height of the salt in the salt chamber reduced but also the contact between the salt and the HTS or thermocouples is no longer necessarily given. Voids are formed in the salt volumes that additionally reduce heat conduction in the material. Additionally, the temperature difference is smaller, so that the driving force behind the heat transfer is weaker.

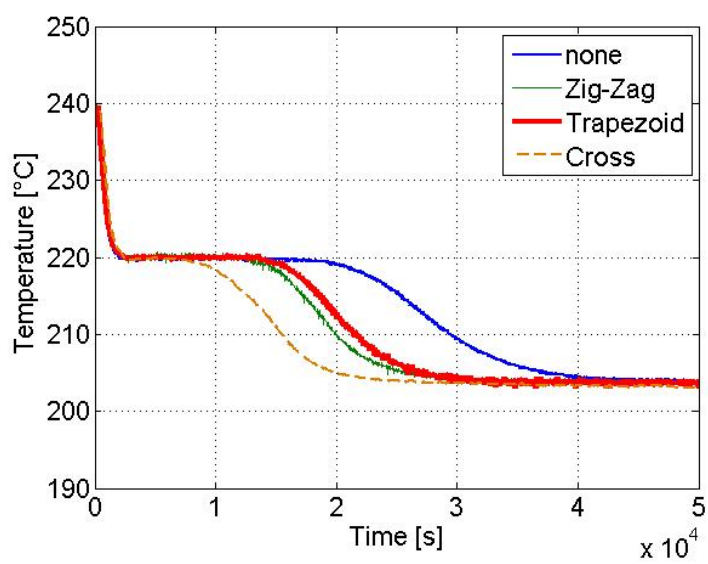

(a)

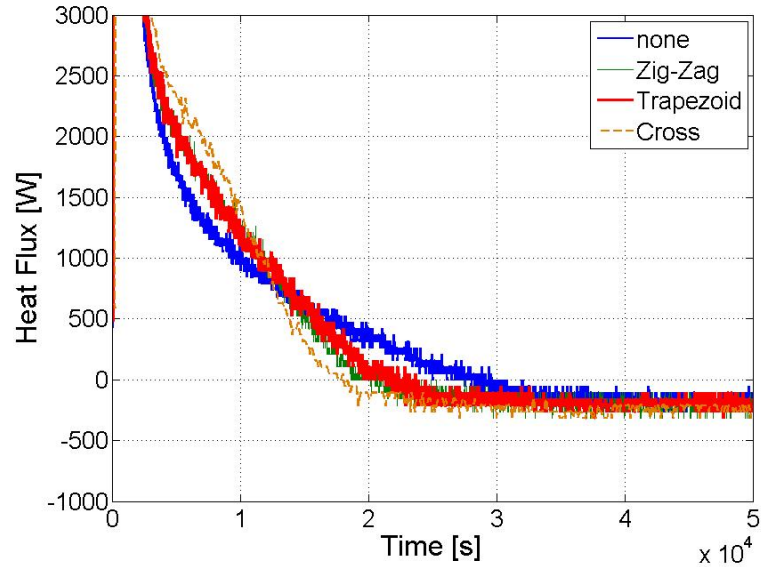

(b)

FIGURE 2: Results for discharging with a volumetric flow of $2 \mathrm{~m}^{3} / \mathrm{h}$ and a temperature difference about the PCM melting point of $\pm 18 \mathrm{~K}$ for set-ups without a HTS and with each of the three tested structures. (a) Temperature measurements in the PCM. (b) Heat flux across the heat transfer medium between inlet and outlet of the storage.

In comparison to each other, the Cross structure discharges much more quickly than the Zig-Zag and Trapezoid structures, as well as faster than the experiments without a HTS. As explained above, a reason for introducing the HTS is to improve the heat transfer during discharging. In a PCM storage unit without HTS, energy is transferred 
very quickly at the beginning of discharging and this transfer decreases quickly as a solid layer of salt forms on the heat transfer surface. This is shown in Fig. 2 (b) in the solid blue line. In this diagram, the heat flux between the inlet and outlet of the heat transfer medium of the storage unit are plotted over time. This is calculated from the flow measurements and inlet and outlet temperatures of the heat transfer medium. The y-axis is zoomed to cut off heat flux during the sensible heat transfer, as this is not of interest in this analysis. In comparison to the experiments without a HTS, the measurements with the Zig-Zag and Trapezoid have a flatter slope, showing that, at this small scale, a change in the heat flux was measured. The slope of the Cross structure is flatter yet. As a similar amount of energy can be discharged from the storage in each case, the flatter slope logically correlates to the faster phase change times shown in Fig. 2 (a).

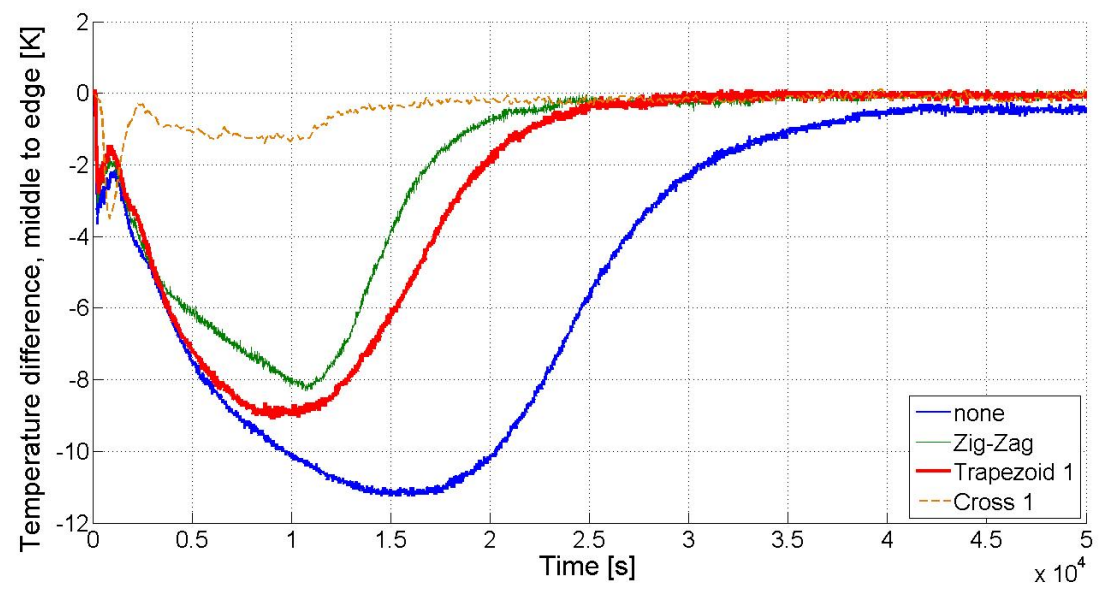

FIGURE 3: Temperature difference between the inner/middle thermocouple and an average of the edge thermocouples during discharging of the storage unit with a temperature range of $\pm 18 \mathrm{~K}$ and a volumetric flow rate of $2 \mathrm{~m}^{3} / \mathrm{h}$ for experiments without HTS and with each of the three tested.

As there is intentionally little temperature difference between the inlet and outlet of the heat transfer fluid in order to simulate a phase changing heat transfer fluid, determining the power level of the storage unit while accounting for losses to the environment is difficult. To better show the changes in power level, the change in the difference between the temperature of the storage material at the middle and edge of a chamber gives additional insight into this power level, however, as a flat curve indicates that heat is transferred quickly into the interior of the PCM mass. In Fig. 3, the temperature difference between the edge thermocouples and the thermocouple furthest from a HTS are plotted for the discharging experiments discussed above, again for the $\pm 18 \mathrm{~K}$ temperature range. This shows clearly that the temperature difference in the Cross experiments remains very small throughout the discharge, showing that the energy is transferred out of the storage effectively over the entire discharge. In the experiments without HTS, on the other hand, the temperature difference between the middle and edge of the chamber is very large, showing that the heat transfer through the solidifying salt is very slow.

These data were analyzed for the various parameter combinations. In Fig. 4, the phase change times for various experiments are plotted on the $\mathrm{x}$-axis. The phase change time is determined as the time during which the temperature is constant, and disregards the sensible heat transfer sections prior to and post phase change. The results shown here are averages of the multiple cycles measured for each parameter combination. The results are plotted for all three temperature ranges and for the three structures as well as experiments without a structure. On the left-hand y-axis, the masses of the structures for each of the experiments are plotted. The masses plotted here are the masses of the HTS inserted into one of the middle, larger chambers. The three dashed lines and the solid markers refer to this axis.

On the right-hand y-axis, the times are plotted referring to the surfaces areas for each of these structures, referring to the three solid lines and the hollow markers. For each of the structures, the masses and surface areas are obviously constant for the temperature range parameter variations. This comparison is shown in one diagram in order to compare how these two factors correlate with the phase change time. As these masses and surface areas refer to the increased mass of the HTS, they are both zero for the experiments without a HTS.

As expected, the phase change time increases with a decrease in both surface area and mass of the inserted HTS. The effect of the HTS is greater with decreasing temperature difference, shown by the decreasing slope of the lines from left to right in Fig. 4. This correlation between phase change time and weight or surface area is most interesting 
when analyzing the data for the Zig-Zag and Trapezoidal structures. Here, the correlation is not as constant. At a temperature difference of $\pm 18 \mathrm{~K}$ (red dash-dot-dot and green midweight lines), for example, there is almost no difference between the phase change times (compare 206 to 208 minutes). This shows that the Zig-Zag structure has a more optimal use of material than the Trapezoid structure. These data also show that the phase change time (correlating to the power of the storage unit, as long as capacity is constant) can be adapted according to the available temperature differences in the industrial process in which the storage unit is to be integrated.
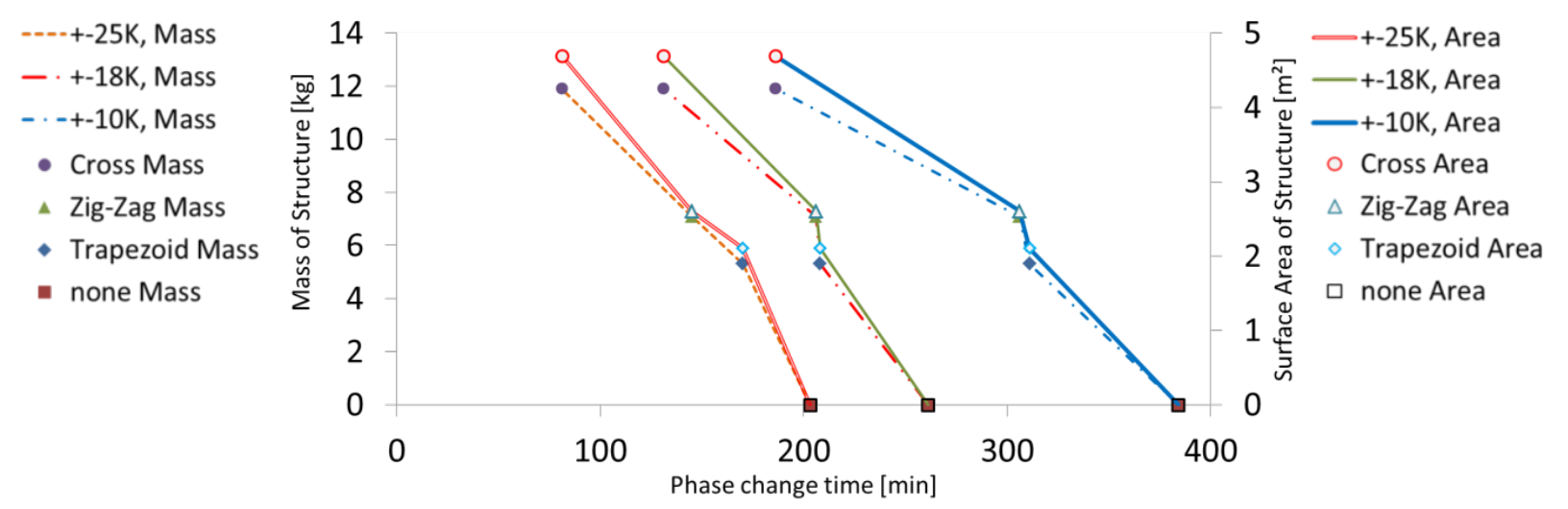

FIGURE 4: Phase change times for the three HTS and without versus the HTS masses and surfaces areas.

Since the HTS masses are different depending on the design and the fill level of the liquid PCM in the storage unit was kept constant throughout all of the experiments, the capacity of the storage unit varied with the physical setups. This naturally also affects the phase change time of the storage units. By dividing the pure latent heat capacity of the storage by the phase change time of the storage unit, the average power for the different temperature ranges and HTS are calculated. These are plotted in Fig. 5, again over the HTS masses and surface areas. This representation of the data clearly shows that the impact of the HTS greatly increases the average power achievable in a latent heat storage unit, and more clearly shows how the Zig-Zag and Cross structures greatly improve the average power, especially at larger temperatures differences. In comparison to the Zig-Zag structure, the Cross structure has a comparatively poor ratio of surface area to weight at $0.394 \mathrm{~m}^{2} / \mathrm{kg}$. This is similar to the Trapezoid structure with $0.397 \mathrm{~m}^{2} / \mathrm{kg}$, but higher than the Zig-Zag at $0.368 \mathrm{~m}^{2} / \mathrm{kg}$. This ratio should be optimized in future work, while maintaining manufacturing possibilities.

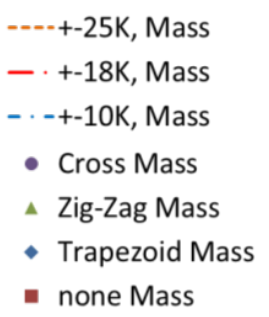

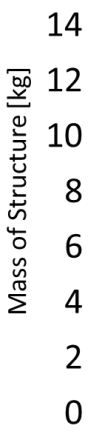

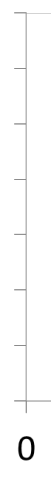

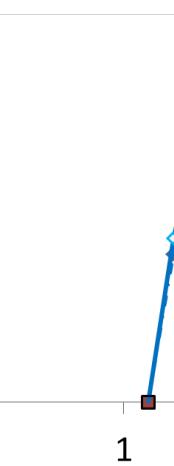




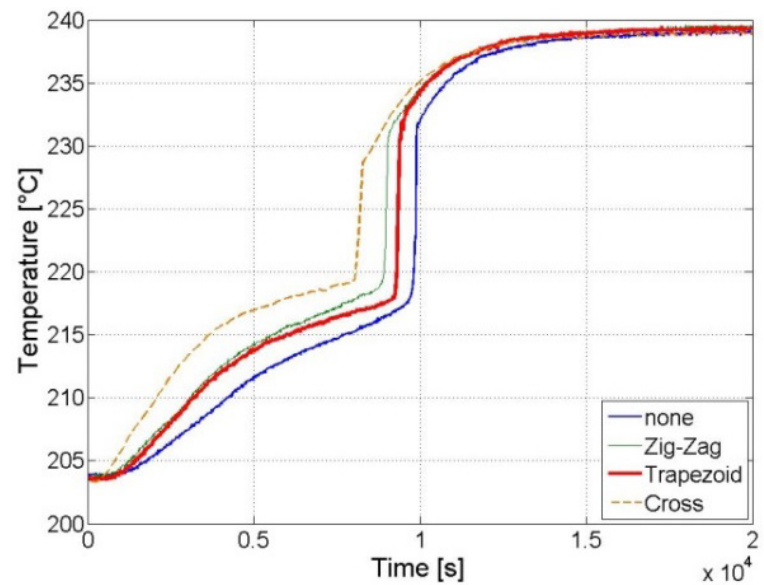

(a)

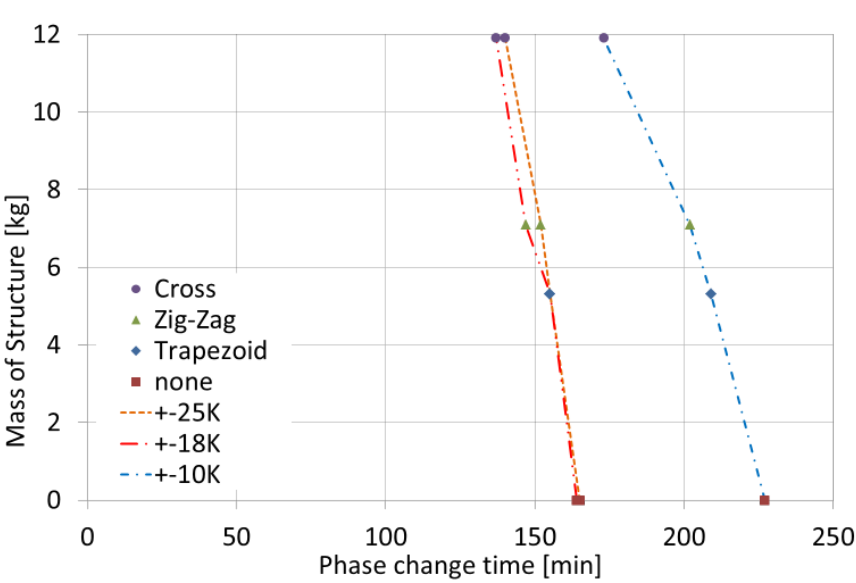

(b)

FIGURE 6: Charging cycle at $2 \mathrm{~m}^{3} / \mathrm{h}$, results for experiments with each of the three HTS and without (a) showing a temperature difference about the PCM melting point of $\pm 18 \mathrm{~K}$ over time and (b) for all time ranges versus the HTS masses.

The HTS masses over the phase change times for the experiments at all three temperature ranges at a volumetric flow rate of $2.0 \mathrm{~m}^{3} / \mathrm{h}$ are plotted in Fig. 6 (b). These show that neither the temperature difference nor the HTS are as influential during charging as during discharging. The greatest variation is shown at the low temperature difference $( \pm 10 \mathrm{~K})$, where the Cross structure finishes charging about $24 \%$ faster than without any HTS.

\section{CONCLUSIONS}

Experimental results for experiments in a flat plate latent heat storage unit for high temperature applications are shown with various heat transfer structures tested. These results have proven the feasibility of the design concept and show that the design concept has an adaptable phase change time and power level for various application requirements. This allows for the integration of this storage concept into solar thermal process applications, where the system parameters vary from process to process significantly. A simulation model integrating these results to calculate storage properties with various conductive structures is in development. Further development of the design for better heat transfer from the heat transfer fluid to the storage material is in process, as well as for adapting the concept for further flexibility in integration parameters.

\section{ACKNOWLEDGMENTS}

The authors would like to thank Christina Schmitt for her data preparation work of this research. Portions of this research were conducted by the author Torsten Klemm while he was a thesis student at DLR. His current affiliation is with the Fraunhofer Institute for Manufacturing Technology and Advanced Materials in Dresden, Germany.

\section{REFERENCES}

1. D. Laing, T. Bauer, N. Breidenbach, B. Hachmann and M. Johnson, Appl. Energy, 109(2), 497-504 (2013).

2. M. Johnson, T. Grünberger and M. Haas, "Verbundvorhaben PROSPER-plus: Weiterentwicklung und anwendungsnahe Erprobung der PROSPER-Latentspeicher-Technologie in der Baustoff Produktion" : Projektabschlussbericht ; Berichtszeitraum: 1. November 2008 - 31. Mai 2011. Doi:10.2314/GBV:72753386X.

3. W. D. Steinmann and D. Laing, J Sol Energy Eng Trans ASME, 132(2), 021003-021003-5 (2010). Doi:10.1115/1.4001465.

4. M. Johnson, M. Fiss, T. Klemm and M. Eck, "Test and analysis of a flat plate latent heat storage design" in Energy Procedia, 57, 662-671 (2014). 
5. T. Bauer, D. Laing and R. Tamme, "Overview of PCMs for concentrated solar power in the temperature range 200 to $350^{\circ} \mathrm{C}$," in Advances in Science and Technology, Vol. 74 (2010) 272-7, doi:10.4028/www.scientific.net/AST.74.272

6. J. Vogel, M. Johnson, M. Eck and D. Laing, "Numerical Analysis of natural convection in a latent heat thermal energy storage system containing rectangular enclosures," in Eurotherm Seminar 99 - Advances in Thermal Energy Storage Systems, May 28-30 2014 in Lleida, Spain, 1-10 (2014). Internet access: elib.dlr.de/89856. 